\title{
To the future of aged society: "Public health and nutrition".
}

\author{
Yu-Ming Chen* \\ Assistant Professor, Master Program of Long-Term Care in Aging, Kaohsiung Medical University \\ Research Fellow, Department of Medical Research, Kaohsiung Medical University Hospital
}

Accepted on January 29, 2018

\section{Editorial}

For the $21^{\text {st }}$ century, $\mathrm{WHO}(2002)$ has proposed a proposal of "sustainable and healthy city" to promote a global strategy for universal health in the future, that remind us must notice the importance of the sustainability of social development and should shape the structure of the overall health environment. Therefore, universal health should be determined from various perspectives of social structure. So, these issues must be considered more in the future:

- Equity of healthcare system

- Community participation

- Health promotion

- Interdisciplinary cooperation

- Strengthens primary care

- International cooperation

That is important missions for public health and health science researchers. With the advanced development of global social, economic and living environment, we can see the rapid progress of medical technology, the development of the public health system and the improvement of human nutrition intake, have led to improvement of the quality of life of people around the world, The gradual extension of life expectancy also makes people's retirement life much longer. Although this is an important progress and achievement of public health in the world, that also brought some new problems to us. For example, the longer average life expectation also prolongs many elderly people suffering from chronic diseases and physical and mental disability that have increased the possible burden of caregiver and long-term resource expenditures of the state, society and families. So, the improvement of long-term care service system for senior citizens, the innovation of long-term care service model and connotation, the chronic modern civilized illness and mental health, holistic public health nutrition strategy, then became the $21^{\text {st }}$ century emerging research focus.

Despite of these progresses of global public health and nutrition, but we still could see some difficult issues need us to seek for solution now.

\section{Efficiency and increasing cost of healthcare system}

because of more and more healthcare needs derived from increasing aged population, increasing costs of medical services and new drug development, many developed countries faced higher and higher costs of healthcare system for decades, but quality of healthcare seemed didn't increase as well, many medical institutions purchased a lot of expensive clinical equipment and instruments, healthcare services became more and more expensive too, but the improvement of health condition for people were not as good as our imagination, even in some developing countries, the working conditions of medical staff have not significantly improved. Perhaps we could ask and do some researches to make a better world. Is there some unnecessary purchasing, or wastes of resources, inappropriate distribution of resources, over-commercialization, or inappropriate mode of cooperation? That's the first key issue we have to figure out to grow a sustainable healthcare system to face the future of aged society.

\section{The fairness and justice of medical service}

In a globalization era and a liberalism capitalism free market of $21^{\text {st }}$ century, when we mention of acquiring quality healthcare services, those who are economically disadvantaged usually means that they are also those who are underprivileged in healthcare and nutrition taking. There are various medical policy models and funding modules around the world, how to balance the differences of human rights and citizenship for medical services and healthcare of the under classes with public health programs, medical policies and clinical services will be the key issue for a developed future.

\section{Regional differences for healthcare}

Many developed and developing countries faced the urbanization of a concentrated urban population, distributions of capacity of medical care delivery systems are very different between rural and urban areas. Because the metropolitan areas are usually quiet rich in economic, culture, education and medical training resources, most of healthcare staff prefer to be employed and owners also prefer to operate institutions in the metropolitan areas, make the gap of resources between urban and rural worse. How to adjust such imbalances will be an important challenge in the future.

\section{Cross-culture and healthcare research}

There are a lot of different healthcare model around the world, every model has each own specialty and advantage, to research different values and ideal of various healthcare system from various countries, could help us to understand their social development, social norms, ideologies, culture and views of national development that reflect their philosophy of society and politics. Healthcare system of a state usually constructed from its' experience of history, culture, religion, economic status, political ideology, social organization, average education level, living standard, welfare attitude, role of government and so on. To study different institutions and programs from various healthcare model, could help us to promote the exchange of 
experiences between different countries and avoid our own blind points, and seek for new possibilities to reform our system.

To sum up, there are some trends of healthcare system reform in developed countries that may influence the direction of healthcare policy making:

- Cost control and efficiency management for healthcare system

- Balance class and religion differences of healthcare service systems and policies

- Interdisciplinary and cross-culture global research cooperation.

Therefore, the academic research community and health care professions must adjust their focus on health science. We need to pay more attention to pay more attention to maintain the long-term health of human beings from the social development structure and state policies, to research practical models of public health and nutrition, and the long-term care of human rights in the aging society. How to make every independent individual in the society to obtain their appropriate health care and nutritional support for life, to practice the rights of freedom, human rights, dignity and self-realization that enjoyed by each individual in universal values, is the current focus of public health and nutrition scholars to research nowadays. Therefore, we started this journal for the above concerns, and hope to open up new research ideas and directions to these concerns. The editorial board of "Public Health and Nutrition" hereby hopes to invite all public health and nutrition experts to contribute your intelligence and research outcomes on our journal. Public Health and Nutrition, is a platform for interdisciplinary research and study. It is our belief that we would be able to work together to protect quality and human rights of healthcare through interdisciplinary discussion and dialogue.

\section{References}

1. World Health Organization.2002.How to Make Cities Healthier. WHO website: http:/www.euro.who.int/en/ health-topics/environment-and-health/urban-health/ activities/healthy-cities.

\section{*Correspondence to:}

Yu-Ming Chen, Ph.D.

Assistant Professor

Master Program of Long-Term Care in Aging

Kaohsiung Medical University, Taiwan

Tel: 886-7-312-1101, ext. 2631

E-mail: yumingchen@kmu.edu.tw 